\title{
Totally real minimal submanifolds in a quaternion projective space
}

\author{
by XIMIN LiU (Tianjin)
}

\begin{abstract}
We prove some pinching theorems with respect to the scalar curvature of 4-dimensional conformally flat (concircularly flat, quasi-conformally flat) totally real minimal submanifolds in $Q P^{4}(c)$.
\end{abstract}

1. Introduction. A quaternion Kähler manifold [3] is defined as a $4 n$-dimensional Riemannian manifold whose holonomy group is a subgroup of $\operatorname{Sp}(1) \cdot \operatorname{Sp}(n)$. A quaternion projective space $Q P^{n}(c)$ is a quaternion manifold with constant quaternion sectional curvature $c \geq 0$.

Let $M$ be an $n$-dimensional Riemannian manifold and $J: M \rightarrow Q P^{n}(c)$ an isometric immersion. If each tangent 2-subspace of $M$ is mapped by $J$ into a totally real plane of $Q P^{n}(c)$, then $M$ is called a totally real submanifold of $Q P^{n}(c)$. Funabashi [2], Chen and Houh [1] and Shen [4] studied this class of submanifolds and got many interesting curvature pinching theorems. The purpose of this paper is to give some pinching theorems with respect to the scalar curvature of 4-dimensional conformally flat (concircularly flat, quasi-conformally flat) totally real minimal submanifolds in $Q P^{4}(c)$.

2. Preliminaries. We give here a quick review of basic formulas for totally real submanifolds in a quaternion Kähler manifold; for details see [1].

Let $(N, g)$ be a $4 m$-dimensional quaternion Kähler manifold with quaternion structure $I, J$ and $K$ satisfying

$$
I J=K, \quad J K=I, \quad K I=J, \quad I^{2}=J^{2}=K^{2}=-1 .
$$

For a unit vector $X$ on $N$, let $Q(X)$ denote the 4-plane spanned by $X$, $I X, J X$ and $K X$, which is called the quaternion-section determined by $X$. Any 2-plane in a quaternion-section is called the quaternion-section-plane,

1991 Mathematics Subject Classification: 53C40, 53C42.

Key words and phrases: totally real submanifold, quaternion projective space, curvature pinching. 
whose sectional curvature is called the quaternion sectional curvature. For any two vectors $X$ and $Y$ on $N$, if $Q(X)$ and $Q(Y)$ are mutually orthogonal, the 2-plane spanned by $X$ and $Y$ is called a totally real plane of $N$. It is well known that $(N, g)$ has constant quaternion curvature $c$ if and only if the curvature $\bar{R}$ of $N$ is of the following form:

$$
\begin{aligned}
\bar{R}(X, Y) Z= & \frac{c}{4}(g(Y, Z) X-g(X, Z) Y+g(I Y, Z) I X-g(I X, Z) I Y \\
& +2 g(X, I Y) I Z+g(J Y, Z) J X-g(J X, Z) J Y \\
& +2 g(X, J Y) J Z+g(K Y, Z) K X \\
& -g(K X, Z) K Y+2 g(X, K Y) K Z) .
\end{aligned}
$$

Let $M$ be an $n$-dimensional Riemannian manifold and $J: M \rightarrow N$ an isometric immersion. If each tangent 2-plane of $M$ is mapped by $J$ into a totally real plane in $N$, then $M$ is called a totally real submanifold of $N$.

In the following, let $Q P^{n}(c)$ denote a $4 n$-dimensional quaternion projective space with constant quaternion sectional curvature $c \geq 0$. Let $M$ be an $n$-dimensional totally real submanifold in $Q P^{n}(c)$ with $n \geq 2$. We choose a local field of orthonormal frames in $Q P^{n}(c)$ :

$$
e_{1}, \ldots, e_{n} ; e_{I(1)}=I e_{1}, \ldots, e_{I(n)}=I e_{n} ; e_{J(1)}=J e_{1}, \ldots, e_{K(n)}=K e_{n}
$$

in such a way that, restricted to $M, e_{1}, \ldots, e_{n}$ are tangent to $M$. We will use the following convention on the range of indices unless otherwise stated:

$$
\begin{gathered}
A, B, C, \ldots=1, \ldots, n, I(1), \ldots, I(n), J(1), \ldots, K(n) ; \\
i, j, k, \ldots=1, \ldots, n ; \\
u, v, \ldots=I(1), \ldots, K(n) ; \\
\phi=I, J \text { or } K .
\end{gathered}
$$

Let $\omega^{A}$ and $\omega_{B}^{A}$ be the dual frame field and the connection forms with respect to the frame field chosen above. Then the structure equations of $Q P^{n}(c)$ are

$$
\begin{aligned}
& d \omega^{A}=-\sum \omega_{B}^{A} \wedge \omega^{B}, \quad \omega_{B}^{A}+\omega_{A}^{B}=0, \\
& d \omega_{B}^{A}=-\sum \omega_{C}^{A} \wedge \omega_{B}^{C}+\frac{1}{2} \sum \bar{R}_{A B C D} \omega^{C} \wedge \omega^{D} .
\end{aligned}
$$

Restricting these forms to $M$, we have

$$
\omega^{u}=0, \quad \omega_{i}^{u}=\sum h_{i j}^{u} \omega^{j}, \quad h_{i j}^{u}=h_{j i}^{u}, \quad h_{j k}^{\phi(i)}=h_{k i}^{\phi(j)}=h_{i j}^{\phi(k)} .
$$

The second fundamental form $\sigma$ of $M$ in $Q P^{n}(c)$ is defined as

$$
\sigma=\sum h_{i j}^{u} \omega^{i} \otimes \omega^{j} \otimes e_{u} .
$$

Its length square is $\|\sigma\|^{2}=\sum\left(h_{i j}^{u}\right)^{2}$. 
If $M$ is minimal in $Q P^{n}(c)$, i.e., trace $\sigma=0$, we have

$$
\varrho=\frac{c}{4} n(n-1)-\|\sigma\|^{2}
$$

where $\varrho$ is the scalar curvature of $M$.

Let $A_{u}$ and $\Delta$ denote the $(n \times n)$-matrix $\left(h_{i j}^{u}\right)$ and the Laplacian on $M$, respectively. We have the following formula [1]:

$$
\begin{aligned}
\frac{1}{2} \Delta\|\sigma\|^{2}= & \left\|\nabla^{\prime} \sigma\right\|^{2}+\sum \operatorname{tr}\left(A_{u} A_{v}-A_{v} A_{u}\right)^{2} \\
& -\sum\left(\operatorname{tr} A_{u} A_{v}\right)^{2}+\frac{c}{4}(n+1)\|\sigma\|^{2} .
\end{aligned}
$$

Since $\sum \operatorname{tr}\left(A_{u} A_{v}-A_{v} A_{u}\right)^{2}=-\sum\left(\sum_{m}\left(h_{k m}^{u} h_{l m}^{v}-h_{k m}^{v} h_{l m}^{u}\right)\right)^{2}$, this together with the equation of Gauss implies

$$
\sum \operatorname{tr}\left(A_{u} A_{v}-A_{v} A_{u}\right)^{2}=-\|R\|^{2}+c \varrho-\frac{n-1}{8} n c^{2} .
$$

Similarly, we have

$$
\sum\left(\operatorname{tr} A_{u} A_{v}\right)^{2}=\|S\|^{2}-\frac{n-1}{2} c \varrho+n\left(\frac{n-1}{4} c\right)^{2}
$$

where $S$ is the Ricci tensor of $M$.

Combining (3)-(6), we obtain

$$
\frac{1}{2} \Delta\|\sigma\|^{2}=\left\|\nabla^{\prime} \sigma\right\|^{2}-\|R\|^{2}-\|S\|^{2}+\frac{n+1}{4} c \varrho .
$$

3. Conformally flat totally real minimal submanifolds. Suppose $M$ is an $n$-dimensional compact oriented totally real minimal submanifold in $Q P^{n}(c)$. If $M$ is conformally flat, then its conformal curvature tensor $C$ [6] satisfies

$$
\begin{aligned}
C(X, Y, Z . W)= & R(X, Y, Z, W)-\varrho(g(X, W) g(Y, Z) \\
& -g(Y, W) g(X, Z)) /(n(n-1)) \\
& -(g(X, W) g(Y, Z)-g(Y, W) G(X, Z) \\
& +g(Y, Z) G(X, W)-g(X, Z) G(Y, W)) /(n-2)=0
\end{aligned}
$$

where $G(X, Y)=S(X, Y)-\varrho g(X, Y) / n$. From (8) we have

$$
\|R\|^{2}=\frac{4}{n-2}\|S\|^{2}-\frac{2 \varrho^{2}}{(n-1)(n-2)} .
$$

Taking the integrals of the both sides of (7) and using (9), by the Green theorem, we have

$$
\int_{M}\left\|\nabla^{\prime} \sigma\right\|^{2} d V=\int_{M}\left(\frac{n+2}{n-2}\|S\|^{2}-\frac{n+1}{4} c \varrho-\frac{2 \varrho^{2}}{(n-1)(n-2)}\right) d V .
$$


On the other hand, by the Gauss-Bonnet theorem, when $n=4$, the Euler number $\chi(M)$ of $M$ is given by

$$
\chi(M)=\frac{1}{32 \pi^{2}} \int_{M}\left(\|R\|^{2}-4\|S\|^{2}+\varrho^{2}\right) d V .
$$

From (9)-(11) we get, when $n=4$,

$$
48 \pi^{2} \chi(M)+\int_{M}\left\|\nabla^{\prime} \sigma\right\|^{2} d V=\int_{M}\left(\frac{2}{3} \varrho^{2}-\frac{5}{4} c \varrho\right) d V .
$$

This yields the following theorem.

Theorem A. Let $M$ be a 4-dimensional compact oriented conformally flat totally real minimal submanifold in $Q P^{4}(c)$. If $M$ has nonnegative Euler number and the scalar curvature $\varrho$ of $M$ is between 0 and $15 c / 8$, then $\varrho$ is 0 or $15 c / 8$.

4. Concircularly flat totally real minimal submanifolds. Suppose $M$ is an $n$-dimensional compact oriented totally real minimal submanifold in $Q P^{n}(c)$. If $M$ is concircularly flat, then its concircular curvature tensor $B$ [5] satisfies

$$
\begin{aligned}
B(X, Y, Z . W)= & R(X, Y, Z, W)-\varrho(g(X, W) g(Y, Z) \\
& -g(Y, W) g(X, Z)) /(n(n-1))=0 .
\end{aligned}
$$

From (13) we have

$$
\|R\|^{2}=2 \varrho^{2} /(n(n-1)) .
$$

Since $M$ is compact and oriented, from (7), (14) and the Green theorem we can obtain

$$
\int_{M}\left\|\nabla^{\prime} \sigma\right\|^{2} d V=\int_{M}\left(\|S\|^{2}+\frac{2 \varrho^{2}}{n(n-1)}-\frac{n+1}{4} c \varrho\right) d V .
$$

When $n=4$, from (11), (14) and (15) we have

$$
32 \pi^{2} \chi(M)+4 \int_{M}\left\|\nabla^{\prime} \sigma\right\|^{2} d V=\int_{M} \frac{11}{6} \varrho\left(\varrho-\frac{30}{11} c\right) d V .
$$

When $\chi(M)$ is nonnegative, we obtain the following theorem.

Theorem B. Let $M$ be a 4-dimensional compact oriented concircularly flat totally real minimal submanifold in $Q P^{4}(c)$. If $M$ has nonnegative Euler number and the scalar curvature $\varrho \geq 0$, then $M$ is totally geodesic.

5. Quasi-conformally flat totally real minimal submanifolds. Let $M$ be an $n$-dimensional compact oriented totally real minimal submanifold 
in $Q P^{n}(c)$. If $M$ is quasi-conformally flat, then its quasi-conformal curvature tensor $H[6]$ satisfies

$$
\begin{aligned}
H(X, Y, Z . W)= & B(X, Y, Z, W)-\mu(g(X, W) G(Y, Z) \\
& -g(Y, W) G(X, Z)+g(Y, Z) G(X, W) \\
& -g(X, Z) G(Y, W))=0
\end{aligned}
$$

where $G(X, Y)=S(X, Y)-\varrho g(X, Y) / n=0$ and $\mu$ is a constant. From (17) we have

$$
\|R\|^{2}=4 \mu^{2}(n-2)\|S\|^{2}+\left(\frac{2}{n-1}-4 \mu^{2}(n-2)\right) \varrho^{2} / n .
$$

From (7), (18) and the Green theorem we get

$$
\begin{aligned}
\int_{M}\left\|\nabla^{\prime} \sigma\right\|^{2} d V= & \int_{M}\left(\left(4 \mu^{2}(n-2)+1\right)\|S\|^{2}\right. \\
& \left.-4 \mu^{2}(n-2)-\frac{2}{n-1} \frac{\varrho^{2}}{n}-(n+1) \frac{c}{4} \varrho\right) d V .
\end{aligned}
$$

When $n=4$, from (11), (18) and (19) we have

$$
\begin{aligned}
32\left(1+8 \mu^{2}\right) \pi^{2} \chi(M)+ & 4\left(1-2 \mu^{2}\right) \int_{M}\left\|\nabla^{\prime} \sigma\right\|^{2} d V \\
& =\int_{M}\left(\varrho-\frac{1-2 \mu^{2}}{11-12 \mu^{2}} 30 c\right) \frac{\varrho}{6}\left(11-12 \mu^{2}\right) d V .
\end{aligned}
$$

Case (I): $\mu^{2}<\frac{8}{61}, \frac{1-2 \mu^{2}}{11-12 \mu^{2}} 30 c>\frac{n\left(2 n^{2}-5 n-1\right)}{2(6 n-1)} c(n=4)$. Then from Theorem 4 of [1], we have

Theorem C. Let $M$ be a 4-dimensional compact oriented quasi-conformally flat totally real minimal submanifold in $Q P^{4}(c)$. If $M$ has nonnegative Euler number and scalar curvature $\varrho \geq 0$, then $M$ is totally geodesic.

Case (II): $\frac{8}{61} \leq \mu^{2} \leq \frac{1}{2}, 1-2 \mu^{2} \geq 0$. Then we have

TheOrem $\mathrm{C}^{\prime}$. Let $M$ be a 4-dimensional compact oriented quasi-conformally flat totally real minimal submanifold in $Q P^{4}(c)\left(\frac{8}{61} \leq \mu^{2} \leq \frac{1}{2}\right)$. If $M$ has nonnegative Euler number and the scalar curvature $\varrho$ of $M$ is between 0 and $\frac{1-2 \mu^{2}}{11-12 \mu^{2}} 30 c$, then $\varrho$ is 0 or $\frac{1-2 \mu^{2}}{11-12 \mu^{2}} 30 c$.

Remark. If $\mu=0, M$ is concircularly flat, then Theorem $\mathrm{C}$ becomes Theorem B. 


\section{References}

[1] B. Y. Chen and C. S. Houh, Totally real submanifolds of a quaternion projective space, Ann. Mat. Pura Appl. 120 (1979), 185-199.

[2] S. Funabashi, Totally real submanifolds of a quaternionic Kaehlerian manifold, Kodai Math. Sem. Rep. 29 (1978), 261-270.

[3] S. Ishihara, Quaternion Kählerian manifolds, J. Differential Geom. 9 (1974), 483500.

[4] Y. B. Shen, Totally real minimal submanifolds in a quaternion projective space, Chinese Ann. Math. Ser. B 14 (1993), 297-306.

[5] K. Yano, Concircular geometry I, Proc. Imp. Acad. Sci. Japan Tokyo 16 (1940), 195-200.

[6] K. Yano and S. Sawaki, Riemannian manifolds admitting a conformal transformation group, J. Differential Geom. 2 (1968), 161-184.

Department of Mathematics

Nankai University

Tianjin 300071

P.R. China
Current address:

Department of Applied Mathematics

Dalian University of Technology

Dalian 116024

P.R. China

E-mail: xmliu@dlut.edu.cn 